Article

\title{
Activated Carbon Supported Hafnium(IV) Chloride as an Efficient, Recyclable, and Facile Removable Catalyst for Expeditious Parallel Synthesis of Benzimidazoles
}

\author{
Xiao-Chong Peng ${ }^{1}$, Shan-Shan Gong ${ }^{1} \mathbb{D}$, De-Yun Zeng ${ }^{1}$, Shu-Wang Duo ${ }^{2, *} \mathbb{C}$ and Qi Sun ${ }^{1, *} \mathbb{C}$ \\ 1 Jiangxi Key Laboratory of Organic Chemistry, Jiangxi Science and Technology Normal University, \\ 605 Fenglin Avenue, Nanchang 330013, Jiangxi, China; pengxiaochong6@126.com (X.-C.P.); \\ gongshanshan@jxstnu.edu.cn (S.-S.G.); zengdeyun8@126.com (D.-Y.Z.) \\ 2 Jiangxi Key Laboratory of Surface Engineering, Jiangxi Science and Technology Normal University, \\ 605 Fenglin Avenue, Nanchang 330013, Jiangxi, China \\ * $\quad$ Correspondence: swduo@imr.ac.cn (S.-W.D.); sunqi@jxstnu.edu.cn (Q.S.); Tel.: +86-791-8380-5183 (Q.S.)
}

Received: 19 March 2020; Accepted: 14 April 2020; Published: 17 April 2020

check for updates

\begin{abstract}
A highly efficient method for parallel synthesis of a diversity of 1,2-disubstituted benzimidazoles from $\mathrm{N}$-substituted phenylenediamines and aldehydes has been developed by using $10 \mathrm{~mol} \% \mathrm{HfCl}_{4}$ on activated carbon $\left(\mathrm{HfCl}_{4} / \mathrm{C}\right)$ as the catalyst. The newly reported $\mathrm{HfCl}_{4} / \mathrm{C}$ catalyst not only mediated fast and clean formation of benzimidazoles but also could be easily removed from the reaction solution and reused up to eight times. Scanning electron microscope (SEM) and thermal desorption studies showed that activated carbon could reversibly adsorb and release $\mathrm{Hf}(\mathrm{IV})$ in ethanol upon cooling and heating, thereby serving as a thermal-controlled solid support.
\end{abstract}

Keywords: benzimidazole; hafnium chloride; catalysis; activated carbon; parallel synthesis

\section{Introduction}

As one of the most important heterocyclic scaffolds, 1,2-disubstituted benzimidazole has been extensively employed in the development of novel pharmaceutical agents and functional materials [1-3]. In contemporary drug discovery, a huge number of benzimidazole derivatives have been synthesized and utilized as anti-infective, anti-inflammatory, antihypertensive, antitumor, antiallergic, antidiabetic, analgesic, and proton pump inhibitory agents [4-7].

In our previous research, we found that the synthesis of 2-aminovinyl benzimidazoles represented a huge challenge to the commonly known synthetic methods for benzimidazoles. To promote the condensation of 1,2-phenylenediamines with $N$-arylated 3-aminoacroleins, both acidic catalysts (e.g., PPA [8] and $\mathrm{BF}_{3} \cdot \mathrm{Et}_{2} \mathrm{O}$ [9]) and oxidative reagents (e.g., DDQ [10], $\mathrm{MnO}_{2}$ [11], I2 [12], and Oxone [13]) were tested. However, none of these methods afforded the desired 2-aminovinyl benzimidazoles, because the conjugation of electron-donating aniline significantly lowered electropositivity of the carbonyl in 3-aminoacroleins. Surprisingly, we found that the non-toxic and inexpensive Group IVB transition metal salts, such as $\mathrm{ZrOCl}_{2} \cdot 8 \mathrm{H}_{2} \mathrm{O}, \mathrm{ZrCl}_{4}$ [14], and $\mathrm{Cp}_{2} \mathrm{ZrCl}_{2}$ [15], exhibited dramatic catalytic effects on the formation of 2-aminovinyl benzimidazoles due to their strong activation capability on carbonyl group. Yin and Gao also reported that the metallocenes of Ti(IV) [16] and $\mathrm{Zr}(\mathrm{IV})$ [17] were highly efficient Lewis acids for other carbonyl-transformation reactions. In recent years, $\mathrm{HfCl}_{4}$, another closely related Group IVB transition metal salt, was revealed to possess even superior activities in many metal Lewis acid-catalyzed reactions [18-20], especially those involving carbonyl activation [21-24]. In addition, it is noteworthy that $\mathrm{ZrOCl}_{2} \cdot 8 \mathrm{H}_{2} \mathrm{O}, \mathrm{ZrCl}_{4}$, and $\mathrm{HfCl}_{4}$ showed 
high catalytic activity in the synthesis of 2-substituted benzimidazoles from 0 -phenylenediamines and orthoesters [25]. As equivalents of carboxylic acids, orthoesters are distinct from aldehyde. The condensation of $o$-phenylenediamines with orthoesters does not require an extra oxidation step to form benzimidazoles as that of $o$-phenylenediamines with aldehydes. Though aldehydes are more favorable starting materials compared to orthoesters due to their much higher commercial availability, the application of $\mathrm{HfCl}_{4}$ as a catalyst for the reaction of $o$-phenylenediamines and aldehydes has never been explored before.

Inspired by these precedent reports, we extended our research to utilize $\mathrm{HfCl}_{4}$ as a strong carbonyl-activating catalyst to promote the condensation of $N$-substituted $o$-phenylenediamines and aldehydes for expeditious synthesis of 1,2-disubstituted benzimidazoles. In this paper, we report the first utilization of $\mathrm{HfCl}_{4}$ on activated carbon $\left(\mathrm{HfCl}_{4} / \mathrm{C}\right)$ as a novel, efficient, recyclable, and easily removable catalyst for parallel synthesis of a diversity of 1,2-disubstituted benzimidazoles. The scanning electron microscope (SEM) and thermal desorption data elucidated that $\mathrm{HfCl}_{4}$ adsorbed on activated carbon could be partially released in refluxing ethanol and efficiently redeposited on activated carbon upon cooling to ambient temperature.

\section{Results and Discussion}

In the preliminary experiments, $N$-phenyl-o-phenylenediamine $(\mathbf{1}\{1\})$ and benzaldehyde $(\mathbf{2}\{1\})$ in a 1:1 ratio were reacted in the presence or absence of $10 \mathrm{~mol} \%$ Group IVB metal catalysts in ethanol at room temperature without inert gas protection. The data listed in Table 1 showed that the control reaction without catalyst was sluggish $(96 \mathrm{~h})$ and afforded product $3\{1,1\}$ in $81 \%$ yield. $\mathrm{TiCl}_{4}$ shortened the reaction time to $20 \mathrm{~h}$, but the yield of $3\{1,1\}$ was low due to the formation of polar byproducts. All $\mathrm{Zr}(\mathrm{IV})$-catalyzed reactions went to completion in $16-20 \mathrm{~h}$ with $89 \%-92 \%$ yields of $3\{1,1\}$. This result was in accordance with a previous report on $\mathrm{ZrOCl}_{2} \cdot 8 \mathrm{H}_{2} \mathrm{O}$-catalyzed benzimidazole synthesis under solvent-free conditions [26]. Interestingly, $\mathrm{HfCl}_{4}$-catalyzed reaction was remarkably faster (12 h) and higher-yielding (96\%). It is noteworthy that when the amount of $\mathrm{HfCl}_{4}$ was reduced to $5 \mathrm{~mol} \%$, the reaction time was prolonged to $16 \mathrm{~h}$, but the product yield was not affected. Further experiments showed that the catalytic effect was drastically diminished when the amount of $\mathrm{HfCl}_{4}$ was decreased below $3 \mathrm{~mol} \%$.

Table 1. The effect of catalyst on the synthesis of benzimidazole $3\{1,1\}$.

\begin{tabular}{cccc} 
& & \\
\hline Entry & Catalyst & Reaction Time (h) & Isolated Yield of 3\{1,1\} (\%) \\
\hline 1 & $\mathrm{No}_{1\{1\}}$ & 120 & 82 \\
2 & $\mathrm{TiCl}_{4}$ & 20 & 67 \\
3 & $\mathrm{ZrOCl}_{2} \cdot 8 \mathrm{H}_{2} \mathrm{O}$ & 20 & 89 \\
4 & $\mathrm{ZrCp}_{2} \mathrm{Cl}_{2}$ & 20 & 90 \\
5 & $\mathrm{ZrCl}_{4}$ & 16 & 96 \\
6 & $\mathrm{HfCl}_{4}$ & 12 & 90 \\
\hline
\end{tabular}

As expected, increasing temperature significantly accelerated the reaction rate (Table 2, entries 1-4). When the reaction with $5 \mathrm{~mol}_{\%} \mathrm{HfCl}_{4}$ was performed in refluxing ethanol, the reaction time was shortened to only $1 \mathrm{~h}$ without affecting the yield of $3\{1,1\}$. The solvent effect was also investigated (Table 2, entries 5-8). The $\mathrm{HfCl}_{4}$-catalyzed reactions proceeded with comparable yields in DMF, $\mathrm{CH}_{3} \mathrm{CN}$, and dichloroethane (DCE) except that the reaction in DCE was much slower. The reaction in THF generated a significant amount of polar byproducts and required $6 \mathrm{~h}$ to complete. It was interesting to observe that the reaction solution immediately turned into orange color upon addition 
of $\mathrm{HfCl}_{4}$ (Table 2, entry 4). Meanwhile, TLC showed that most of $\mathbf{1}\{1\}$ and $2\{1\}$ starting materials disappeared quickly and were converted into the corresponding colored imine and benzimidazoline intermediates in the presence of $\mathrm{HfCl}_{4}$. In contrast, the formation of the colored intermediates was much slower without a catalyst. These results indicated that $\mathrm{HfCl}_{4}$ promoted the formation of both imine and benzimidazoline intermediates, which is similar to the catalytic mechanism of $\mathrm{Hf}(\mathrm{IV})$ on the formation of fluorinated benzimidazolines elucidated by NMR tracing data [27]. Subsequently, aerial oxidation of the benzimidazoline intermediate smoothly afforded the desired benzimidazole $3\{1,1\}$ as described in many precedent reports [28-30].

Table 2. The effects of temperature and solvent on the synthesis of $3\{1,1\}$ with $5 \mathrm{~mol} \% \mathrm{HfCl}_{4}$.

\begin{tabular}{ccccc}
\hline Entry & Temperature $\left({ }^{\circ} \mathbf{C}\right)$ & Solvent & Reaction Time (h) & Isolated Yield of 3\{1,1\} (\%) \\
\hline 1 & 20 & EtOH & 16 & 96 \\
2 & 40 & EtOH & 12 & 97 \\
3 & 60 & $\mathrm{EtOH}$ & 4 & 97 \\
4 & 80 & $\mathrm{EtOH}$ & 1 & 97 \\
5 & 80 & $\mathrm{DMF}$ & 1 & 88 \\
6 & 80 & $\mathrm{CH}_{3} \mathrm{CN}$ & 1.5 & 92 \\
7 & 80 & $\mathrm{DCE}$ & 24 & 90 \\
8 & 70 & $\mathrm{THF}$ & 6 & 64 \\
\hline
\end{tabular}

To test the possibility to recycle the catalyst, we loaded the $\mathrm{HfCl}_{4}$ onto a series of activated solid supports $(5 \% \mathrm{w} / \mathrm{w})$. Under the optimized reaction conditions, $10 \mathrm{~mol} \%$ of the supported $\mathrm{HfCl}_{4} \mathrm{was}$ applied as the catalyst. The results listed in Table 3 showed that the catalytic effects of $\mathrm{HfCl}_{4} / \mathrm{C}$, $\mathrm{HfCl}_{4} / \mathrm{Al}_{2} \mathrm{O}_{3}$, and $\mathrm{HfCl}_{4} / \mathrm{K}-10$ montmorillonite were similar, where the $\mathrm{HfCl} \mathrm{H}_{4} / \mathrm{SiO}_{2}$-catalyzed reaction required a longer reaction time $(2 \mathrm{~h})$. However, these supported catalysts exhibited huge differences upon reuse. Compared with the other three supported catalysts whose potencies remarkably decreased in the 2 nd round, $\mathrm{HfCl}_{4} / \mathrm{C}$ showed consistent catalytic activity in terms of both yield and reaction

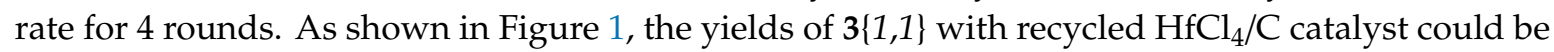
maintained (over 95\%) up to 8 rounds. However, the reaction time was gradually prolonged from 1 to $2 \mathrm{~h}$ in the 5 th to 8 th rounds.

Table 3. The effects of supported $\mathrm{HfCl}_{4}$ catalysts $(10 \mathrm{~mol} \%, 5 \% \mathrm{w} / \mathrm{w})$ on the synthesis of $3\{1,1\}$.

\begin{tabular}{cccc}
\hline Entry & Solid Support & Reaction Time 1st/2nd (h) & Isolated Yield of 3\{1,1\} 1st/2nd (\%) \\
\hline 1 & $\mathrm{SiO}_{2}$ & $2 / 4$ & $92 / 78$ \\
2 & activated carbon & $1 / 1$ & $96 / 96$ \\
3 & $\mathrm{Al}_{2} \mathrm{O}_{3}$ & $1 / 4$ & $93 / 75$ \\
4 & $\mathrm{~K}-10$ montmorillonite & $1 / 4$ & $94 / 79$ \\
\hline
\end{tabular}

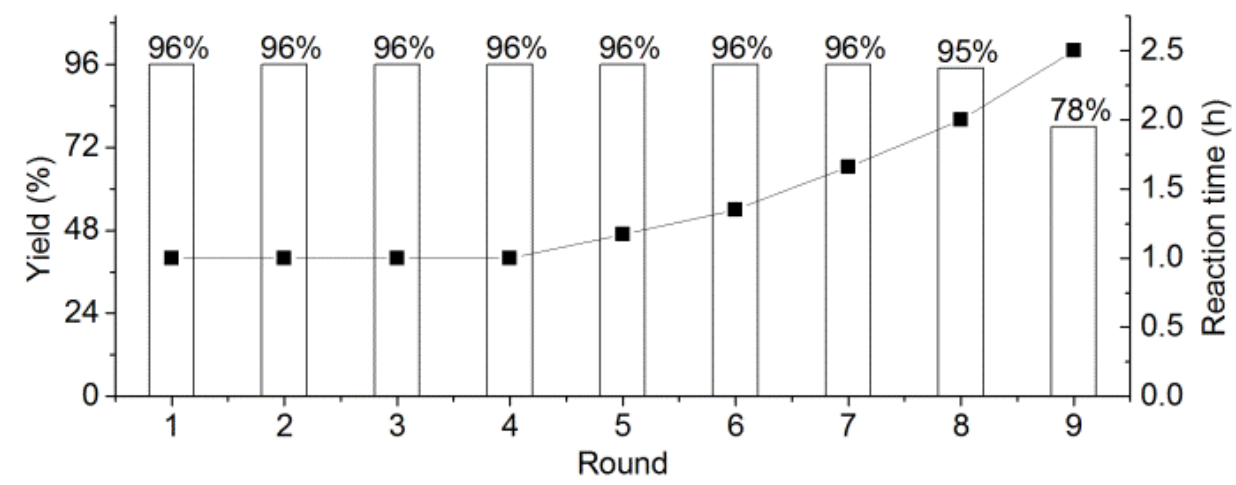

Figure 1. The recyclability of $\mathrm{HfCl}_{4} / \mathrm{C}$ on the synthesis of $3\{1,1\}$. 
To determine how much $\mathrm{HfCl}_{4}$ was released into ethanol as homogeneous catalyst at $80{ }^{\circ} \mathrm{C}$, $\mathrm{HfCl}_{4} / \mathrm{C}(5 \% \mathrm{w} / \mathrm{w}, 500 \mathrm{mg})$ was added to ethanol $(16 \mathrm{~mL})$ and refluxed for $30 \mathrm{~min}$. The solid was filtered while the solution was still at $80{ }^{\circ} \mathrm{C}$. The weight loss data (Table 4) showed that, in the first use, $30 \%$ of $\mathrm{HfCl}_{4}$ desorbed from the surface of activated carbon and was released into the reaction solution. It took 5 times before the $\mathrm{HfCl}_{4}$ was completely washed off. If ethanol was cooled to room temperature before filtration, mimicking the reaction workup procedure, the weight loss was almost negligible after 5 rounds. These results indicated that activated carbon could function as an efficient thermal-controlled sponge that enabled reversible adsorption and release of $\mathrm{HfCl}_{4}$ catalyst in ethanol upon cooling and heating.

Table 4. The ethanol desorption experiments of $\mathrm{HfCl}_{4} / \mathrm{C}$.

\begin{tabular}{ccccccc}
\hline \multirow{2}{*}{ Method Weight Loss (mg) } \\
\cline { 2 - 7 } & 1st & 2nd & 3rd & 4th & 5th & 6th \\
\hline $\mathrm{A}^{\mathrm{a}}$ & 8 & 7 & 5 & 3 & 1 & 0 \\
$\mathrm{~B}^{\mathrm{b}}$ & 0 & 0 & 0 & 0 & 0 & 1 \\
\hline \multicolumn{6}{c}{${ }^{\text {a }}$ Reflux/hot filtration. ${ }^{\mathrm{b}}$ Reflux/cooling/cold filtration. }
\end{tabular}

As depicted in the scanning electron microscope (SEM) images of $\mathrm{HfCl}_{4} / \mathrm{C}$ samples, most $\mathrm{HfCl}_{4}$ initially loaded onto activated carbon appeared as small crystalline-like solids (Figure 2A). After 5 rounds of hot filtration, the surface of the solid support was as clean as that of pure activated carbon. In contrast, the $\mathrm{HfCl}_{4} / \mathrm{C}$ sample, which was filtered after cooling for 5 times still adsorbed $\mathrm{Hf}(\mathrm{IV})$ salt as disordered and amorphous solids (Figure 2B). These results were in good accordance with the thermal desorption experiments mentioned above. Meanwhile, the energy dispersive spectrum (EDS) analysis of the samples confirmed that the solids on the surface of activated carbon were hafnium salts (Figure 2). It is worth noting that the $\mathrm{Cl}$ element almost disappeared after 5 rounds of refluxing/cooling/filtration, indicating that chloride was gradually exchanged to ethoxide upon repeated use. However, the recyclability of the catalyst suggested that the counter ion had relatively less important effect on the catalytic activity.
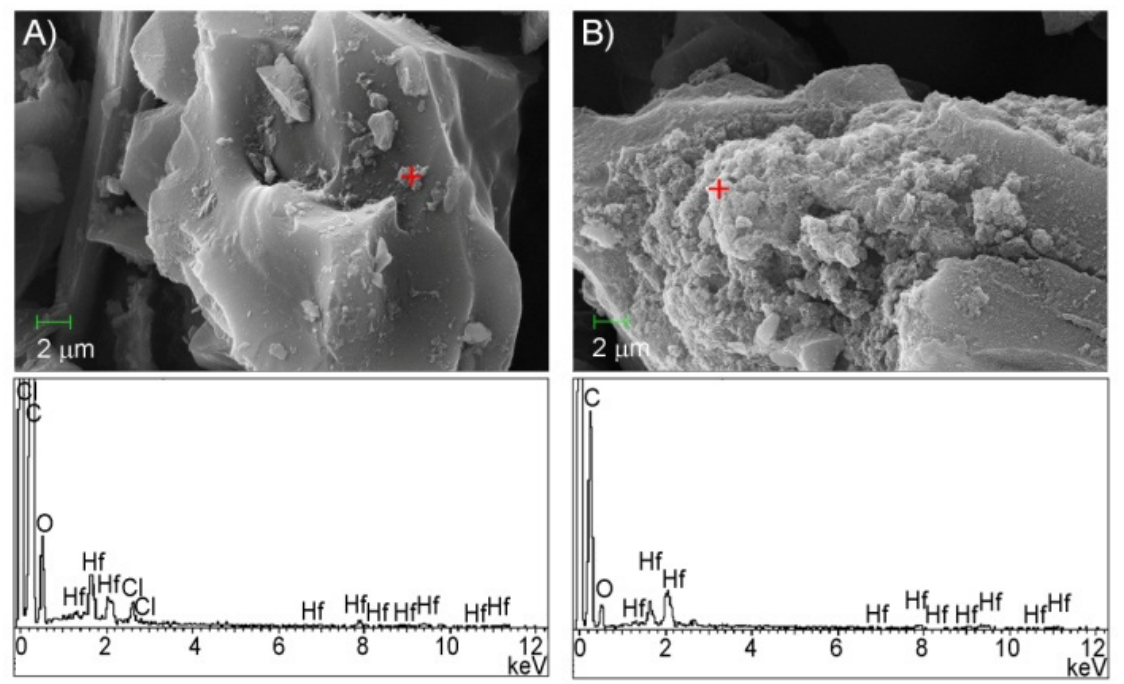

Figure 2. Scanning electron microscope (SEM) images and energy dispersive spectrum (EDS) analysis of $\mathrm{HfCl}_{4} / \mathrm{C}(5 \% \mathrm{w} / \mathrm{w})$ samples. (A) $\mathrm{HfCl}_{4} / \mathrm{C}$ and (B) $\mathrm{HfCl}_{4} / \mathrm{C}$ (reflux/cold filtrate for 5 rounds).

Since $\mathrm{HfCl}_{4}$ was tightly adsorbed on activated carbon at room temperature, we were interested to clarify whether $\mathrm{HfCl}_{4} / \mathrm{C}$ could catalyze the formation of benzimidazole $3\{1,1\}$ in a heterogeneous manner. The experimental result showed that $\mathrm{HfCl}_{4} / \mathrm{C}(10 \mathrm{~mol} \%)$ indeed promoted the formation 
of $3\{1,1\}$ at room temperature. However, as expected, the heterogeneous catalysis $(89 \%, 24 \mathrm{~h})$ was less efficient than the homogeneous catalysis (Table 1, entry 6). These results suggested that, under the refluxing conditions, $\mathrm{HfCl}_{4} / \mathrm{C}$ catalyzed the formation of benzimidazole $3\{1,1\}$ in a combined homogenous/heterogeneous manner.

Other than the high potency and recyclability of $\mathrm{HfCl}_{4} / \mathrm{C}$, another huge advantage of this novel catalyst was that it could be easily removed from the reaction solution without leaving residual metal Lewis acid in the crude product. Therefore, $\mathrm{HfCl}_{4} / \mathrm{C}$ may be applied as an ideal catalyst for expeditious parallel synthesis of benzimidazole derivatives. To prove this point, a diversity of $\mathrm{N}$-substituted $o$-phenylenediamines $(\mathbf{1}\{1-9\})$ and aldehydes $(\mathbf{2}\{1-10\})$ were employed as substrates and total 28 benzimidazoles $(3\{1-9,1-10\})$ were prepared on a parallel synthesizer with $\mathrm{HfCl}_{4} / \mathrm{C}$ as the catalyst in one single batch. The reactions were heated in tightly capped vials at $80^{\circ} \mathrm{C}$ for $1 \mathrm{~h}$. After the reactions were cooled to ambient temperature, $\mathrm{HfCl}_{4} / \mathrm{C}$ was removed by centrifuge, and the supernatants were concentrated to afford the crude products in $97 \%-101 \%$ yields. The purity of crude benzimidazoles (3) was determined to be $91.9 \%-99.0 \%$ by analytical HPLC. Further flash chromatography afforded 28 benzimidazoles (3) in excellent isolated yields ranging from $87 \%$ to $96 \%$ (Figure 3).

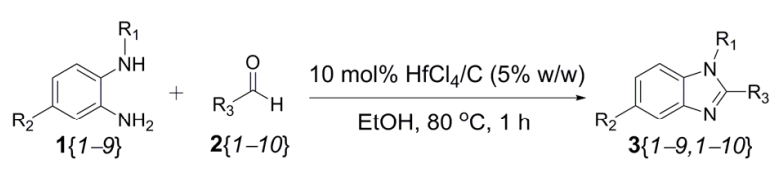

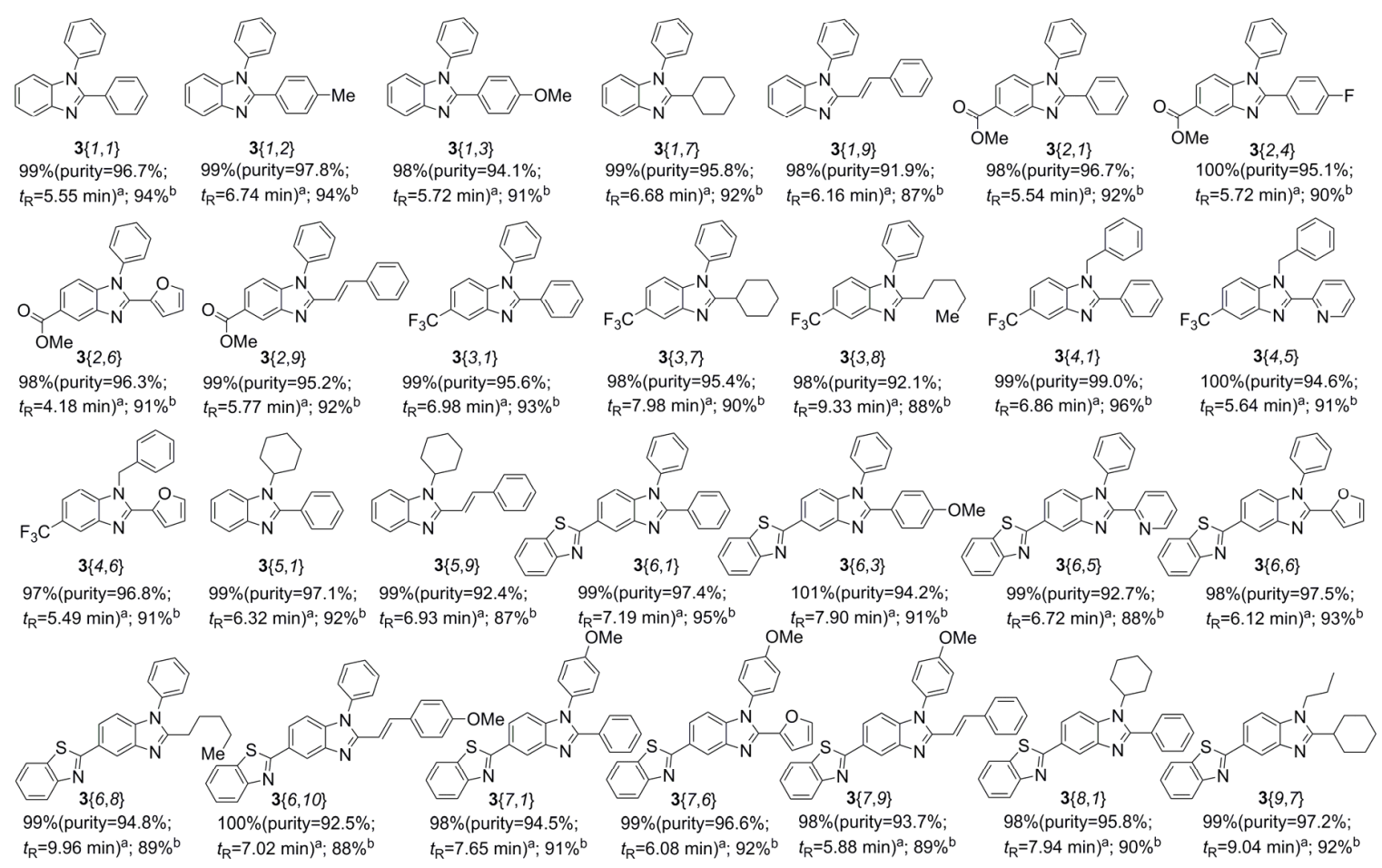

Figure 3. $\mathrm{HfCl}_{4} / \mathrm{C}$-Catalyzed parallel synthesis of benzimidazoles (3). a The yield of crude 3 (purity and retention time determined by analytical HPLC). ${ }^{b}$ The isolated yield of 3 .

\section{Materials and Methods}

\subsection{General Methods}

Chemical reagents (Aladdin, Shanghai, China) were obtained from a commercial supplier. Supported catalysts were prepared according to the methods described below. All reactions were performed in commercial analytical reagent (AR) grade solvents (Zhiyuan Chemicals, Tianjin, China) 
and monitored by thin layer chromatography on plates coated with $0.25 \mathrm{~mm}$ silica gel $60 \mathrm{~F}_{254}$ (Qingdao Haiyang Chemicals, Qingdao, China). TLC plates were visualized by UV irradiation $(254 \mathrm{~nm})$. The parallel synthesis was performed in 28 tightly capped reaction vials $(10 \mathrm{~mL})$ on an aluminum reaction heating block with 48 wells. Melting points were determined with a Thomas-Hoover melting point apparatus and uncorrected (Thomas Scientific, Swedesboro, NJ, USA). NMR spectra were obtained with a Bruker AV-400 instrument (Bruker BioSpin, Faellanden, Switzerland) with chemical shifts reported in parts per million (ppm, $\delta$ ) and referenced to $\mathrm{CDCl}_{3}$. The NMR spectra of new compounds were provided in Supplementary Materials (Figures S1-S38). IR spectra were recorded on a Bruker Vertex-70 spectrometer (Bruker Optics, Billerica, MA, USA). High-resolution mass spectra were reported as $\mathrm{m} / \mathrm{z}$ and obtained with a Dalton micrOTOF-Q II spectrometer (Bruker Daltonics, Billerica, MA, USA). HPLC traces were recorded on an analytical Agilent 1260 Infinity II LC instrument (Angilent Technologies, Palo Alto, CA, USA) equipped with a C18 analytical Angilent Zorbax column $(4.6 \times 150 \mathrm{~mm}, 5 \mu \mathrm{m}$; flow rate $=1.0 \mathrm{~mL} / \mathrm{min} ; 70 \% \mathrm{MeOH}$ in $\mathrm{ddH}_{2} \mathrm{O}$ over $15 \mathrm{~min}$; UV detection at $270 \mathrm{~nm}$ ). The morphology and chemical composition of $\mathrm{HfCl}_{4} / \mathrm{C}$ samples were investigated by a Zeiss Sigma field emission scanning electron microscope (Zeiss microscopy, Jena, Germany).

\subsection{General Procedure for Preparation of $\mathrm{HfCl}_{4} / \mathrm{C}$ Catalyst}

Before impregnation of $\mathrm{HfCl}_{4}$, commercial activated carbon (200 mesh) was pretreated with $30 \%$ $\mathrm{HNO}_{3}$ at $90^{\circ} \mathrm{C}$ for $4 \mathrm{~h}$, washed $\mathrm{ddH}_{2} \mathrm{O}$ until $\mathrm{pH}$ reached 7 , and dried at $120^{\circ} \mathrm{C}$ for $12 \mathrm{~h}$. $\mathrm{HfCl}_{4}(0.5 \mathrm{~g}$, $5 \% \mathrm{w} / \mathrm{w})$ was dissolved in absolute ethanol $(50 \mathrm{~mL})$. Then, pretreated activated carbon $(9.5 \mathrm{~g})$ was added and sonicated for another $30 \mathrm{~min}$ at ambient temperature. Ethanol was then removed under reduced pressure to afford $\mathrm{HfCl}_{4} / \mathrm{C}$.

\subsection{General Procedure for Preparation of Other Supported $\mathrm{HfCl}_{4}$ Catalysts}

Before impregnation of $\mathrm{HfCl}_{4}$, commercial silica gel (300-400 mesh), aluminum oxide (200-300 mesh), and K-10 montmorillonite were heated in an oven at $150{ }^{\circ} \mathrm{C}$ for $24 \mathrm{~h}$. $\mathrm{HfCl}_{4}(0.5 \mathrm{~g}, 5 \%$ $\mathrm{w} / \mathrm{w})$ was dissolved in absolute ethanol $(50 \mathrm{~mL})$. Then, pretreated solid supports $(9.5 \mathrm{~g})$ was added and stirred for $30 \mathrm{~min}$ and sonicated for another $30 \mathrm{~min}$ at ambient temperature. Ethanol was then removed under reduced pressure to afford $\mathrm{HfCl}_{4} / \mathrm{SiO}_{2}, \mathrm{HfCl}_{4} / \mathrm{Al}_{2} \mathrm{O}_{3}$, and $\mathrm{HfCl}_{4} / \mathrm{K}-10$ montmorillonite.

\subsection{General Synthetic Procedure and Characterization of Benzimidazoles}

To a solution of $N$-substituted $o$-phenylenediamines $(0.15 \mathrm{mmol})$ and aldehyde $(0.15 \mathrm{mmol})$ in ethanol $(3 \mathrm{~mL})$ was added $\mathrm{HfCl}_{4} / \mathrm{C}(0.015 \mathrm{mmol}, 5 \% \mathrm{w} / \mathrm{w})$. The reaction was stirred at $80{ }^{\circ} \mathrm{C}$ for $1 \mathrm{~h}$. After the reaction was cooled to ambient temperature, $\mathrm{HfCl}_{4} / \mathrm{C}$ was removed by centrifuge and supernatant was concentrated under reduced pressure. Flash column chromatography on silica gel (petroleum ether:ethyl acetate $=4: 1$ ) afforded benzimidazole in pure form.

1,2-Diphenyl-5-methoxycarbonyl-1H-benzo[d]imidazole $(3\{2,1\})$ : a white solid; mp $147-148{ }^{\circ} \mathrm{C}$. ${ }^{1} \mathrm{H}$ NMR $\left(400 \mathrm{MHz}, \mathrm{CDCl}_{3}\right): \delta 8.60(\mathrm{~s}, 1 \mathrm{H}), 7.98(\mathrm{~d}, J=8.1 \mathrm{~Hz}, 1 \mathrm{H}), 7.61-7.47(\mathrm{~m}, 5 \mathrm{H}), 7.38-7.20(\mathrm{~m}, 6 \mathrm{H})$, $3.95(\mathrm{~s}, 3 \mathrm{H}) \mathrm{ppm} ;{ }^{13} \mathrm{C} \mathrm{NMR}\left(100 \mathrm{MHz}, \mathrm{CDCl}_{3}\right): \delta 167.5,154.1,142.6,140.3,136.5,130.1,129.9,129.5$, 129.0, 128.4, 127.3, 125.2, 124.9, 122.2, 110.2, 52.2 ppm; IR: $v_{\max } 3058,2924,2853,1721,1619,1596,1496$, $1478,1448,1024,982,924,892,827,804,778 \mathrm{~cm}^{-1}$; HRMS (ESI+): $m / z$ calcd for $\mathrm{C}_{21} \mathrm{H}_{17} \mathrm{~N}_{2} \mathrm{O}_{2}[\mathrm{M}+\mathrm{H}]^{+}$ 329.1285; found 329.1280.

2-(4-Fluorophenyl)-1-phenyl-5-methoxycarbonyl-1H-benzo[d]imidazole $(3\{2,4\})$ : a white solid; mp 156-157 ${ }^{\circ} \mathrm{C} .{ }^{1} \mathrm{H}$ NMR $\left(400 \mathrm{MHz}, \mathrm{CDCl}_{3}\right): \delta 8.57(\mathrm{~s}, 1 \mathrm{H}), 7.97(\mathrm{~d}, J=8.5 \mathrm{~Hz}, 1 \mathrm{H}), 7.59-7.47(\mathrm{~m}, 5 \mathrm{H})$, $7.29(\mathrm{~d}, J=7.2 \mathrm{~Hz}, 2 \mathrm{H}), 7.22(\mathrm{~d}, J=8.2 \mathrm{~Hz}, 1 \mathrm{H}), 7.04-6.95(\mathrm{~m}, 2 \mathrm{H}), 3.95(\mathrm{~s}, 3 \mathrm{H}) \mathrm{ppm} ;{ }^{13} \mathrm{C} \mathrm{NMR}(100 \mathrm{MHz}$, $\left.\mathrm{CDCl}_{3}\right): \delta 167.6,165.0,162.5,153.3,142.8,140.5,136.6,131.7,131.6,130.3,129.3,127.9,125.9,125.5,125.2$, 122.4, 115.9, 115.7, 110.3, 52.3 ppm; IR: $v_{\max } 3062,2921,2852,1709,1618,1596,1523,1497,1473,1437$, $1045,987,897,839,802,765 \mathrm{~cm}^{-1}$; HRMS (ESI+): $\mathrm{m} / z$ calcd for $\mathrm{C}_{21} \mathrm{H}_{16} \mathrm{FN}_{2} \mathrm{O}_{2}[\mathrm{M}+\mathrm{H}]^{+} 347.1190$; found 347.1187. 
2-(Furan-2-yl)-1-phenyl-5-methoxycarbonyl-1H-benzo[d]imidazole $(3\{2,6\})$ : a light yellow solid; $\mathrm{mp} 130-131{ }^{\circ} \mathrm{C} .{ }^{1} \mathrm{H}$ NMR $\left(400 \mathrm{MHz}, \mathrm{CDCl}_{3}\right): \delta 8.53(\mathrm{~s}, 1 \mathrm{H}), 7.93(\mathrm{~d}, J=8.8 \mathrm{~Hz}, 1 \mathrm{H}), 7.65-7.56(\mathrm{~m}, 3 \mathrm{H})$, 7.52-7.45 (m, 1H), 7.43-7.36 (m, 2H), $7.07(\mathrm{~d}, J=8.6 \mathrm{~Hz}, 1 \mathrm{H}), 6.39-6.31(\mathrm{~m}, 1 \mathrm{H}), 6.16-6.11(\mathrm{~m}, 1 \mathrm{H}), 3.92$ (s, 3H) ppm; ${ }^{13} \mathrm{C}$ NMR $\left(100 \mathrm{MHz}, \mathrm{CDCl}_{3}\right): \delta 167.5,145.9,144.7,144.1,142.7,140.3,136.0,130.2,129.9$, 127.9, 125.4, 125.2, 122.2, 113.1, 111.7, 109.9, 52.2 ppm; IR: $v_{\max } 3054,2948,2825,1596,1514,1481,1467$, $1458,1043,995,976,928,785 \mathrm{~cm}^{-1}$; HRMS (ESI+): $\mathrm{m} / z$ calcd for $\mathrm{C}_{19} \mathrm{H}_{15} \mathrm{~N}_{2} \mathrm{O}_{3}[\mathrm{M}+\mathrm{H}]^{+}$319.1077; found 319.1082 .

(E)-1-Phenyl-2-styryl-5-methoxycarbonyl-1H-benzo[d]imidazole $(3\{2,9\})$ : a white solid; $\mathrm{mp}$ 195-196 ${ }^{\circ} \mathrm{C} .{ }^{1} \mathrm{H}$ NMR $\left(400 \mathrm{MHz}, \mathrm{CDCl}_{3}\right): \delta 8.54(\mathrm{~s}, 1 \mathrm{H}), 8.02-7.91(\mathrm{~m}, 2 \mathrm{H}), 7.69-7.55(\mathrm{~m}, 3 \mathrm{H})$, 7.51-7.41 (m, 4H), 7.40-7.28 (m, 3H), $7.19(\mathrm{~d}, J=8.8 \mathrm{~Hz}, 1 \mathrm{H}), 6.83(\mathrm{~d}, J=15.6 \mathrm{~Hz}, 1 \mathrm{H}), 3.96(\mathrm{~s}, 3 \mathrm{H}) \mathrm{ppm}$; ${ }^{13} \mathrm{C}$ NMR $\left(100 \mathrm{MHz}, \mathrm{CDCl}_{3}\right): \delta 167.7,152.8,143.1,139.9,138.4,136.0,135.4,130.3,129.5,129.4,129.0$, 127.7, 127.6, 125.6, 124.9, 121.9, 113.7, 109.9, 52.2 ppm; IR: $v_{\max } 3058,2955,2839,1708,1611,1498,1434$, 1015, $797 \mathrm{~cm}^{-1}$; HRMS (ESI+): $\mathrm{m} / z$ calcd for $\mathrm{C}_{23} \mathrm{H}_{19} \mathrm{~N}_{2} \mathrm{O}_{2}[\mathrm{M}+\mathrm{H}]^{+} 355.1441$; found 355.1440 .

1,2-Diphenyl-5-(trifluoromethyl)-1H-benzo[d] imidazole (3\{3,1\}): a white solid; mp $145-146{ }^{\circ} \mathrm{C} .{ }^{1} \mathrm{H}$ NMR $\left(400 \mathrm{MHz}, \mathrm{CDCl}_{3}\right): \delta 8.06(\mathrm{~s}, 1 \mathrm{H}), 7.53-7.36(\mathrm{~m}, 6 \mathrm{H}), 7.32-7.14(\mathrm{~m}, 6 \mathrm{H}) \mathrm{ppm} ;{ }^{13} \mathrm{C}$ NMR $(100 \mathrm{MHz}$, $\left.\mathrm{CDCl}_{3}\right): \delta 154.5,142.6,139.3,136.6,130.2,130.1,129.6,129.4,129.2,128.5,127.4,125.6,123.6,120.3,117.7$, 111.0 ppm; IR: $v_{\max } 3056,2926,2845,1621,1597,1500,1476,1450,1435,1047,1027,978,937,891,860$, 823, $771 \mathrm{~cm}^{-1}$; HRMS (ESI+): $m / z$ calcd for $\mathrm{C}_{20} \mathrm{H}_{14} \mathrm{~F}_{3} \mathrm{~N}_{2}[\mathrm{M}+\mathrm{H}]^{+} 339.1104$; found 339.1109.

2-Cyclohexyl-1-phenyl-5-(trifluoromethyl)-1H-benzo[d]imidazole $(3\{3,7\})$ : a white solid; $\mathrm{mp}$ 93-94 ${ }^{\circ} \mathrm{C} .{ }^{1} \mathrm{H}$ NMR $\left(400 \mathrm{MHz}, \mathrm{CDCl}_{3}\right): \delta 8.06(\mathrm{~s}, 1 \mathrm{H}), 7.65-7.54(\mathrm{~m}, 3 \mathrm{H}), 7.41(\mathrm{~d}, J=8.4 \mathrm{~Hz}, 1 \mathrm{H}), 7.34(\mathrm{~d}$, $J=6.8 \mathrm{~Hz}, 2 \mathrm{H}), 7.11(\mathrm{~d}, J=8.4 \mathrm{~Hz}, 1 \mathrm{H}), 2.77-2.65(\mathrm{~m}, 1 \mathrm{H}), 1.96-1.62(\mathrm{~m}, 6 \mathrm{H}), 1.38-1.11(\mathrm{~m}, 4 \mathrm{H}) \mathrm{ppm}$; ${ }^{13} \mathrm{C}$ NMR $\left(100 \mathrm{MHz}, \mathrm{CDCl}_{3}\right): \delta 161.5,142.3,138.6,135.6,130.3,129.5,127.6,125.2,119.5,117.1,110.5$, 36.4, 32.0, 29.8, 26.2, 25.8 ppm; IR: $v_{\max }$ 3045, 2930, 2855, 1727, 1623, 1598, 1506, 1442, 1042, 926, 883, $809,767 \mathrm{~cm}^{-1}$; HRMS (ESI+): $\mathrm{m} / z$ calcd for $\mathrm{C}_{20} \mathrm{H}_{20} \mathrm{~F}_{3} \mathrm{~N}_{2}[\mathrm{M}+\mathrm{H}]^{+} 345.1573$; found 345.1566 .

2-Pentyl-1-phenyl-5-(trifluoromethyl)-1H-benzo[d]imidazole $(3\{3,8\})$ : colorless oil; ${ }^{1} \mathrm{H}$ NMR $\left(400 \mathrm{MHz}, \mathrm{CDCl}_{3}\right): \delta 8.05(\mathrm{~s}, 1 \mathrm{H}), 7.69-7.52(\mathrm{~m}, 3 \mathrm{H}), 7.43(\mathrm{~d}, J=8.5,1 \mathrm{H}), 7.35(\mathrm{~d}, J=8.1 \mathrm{~Hz}, 2 \mathrm{H}), 7.16(\mathrm{~d}$, $J=8.5 \mathrm{~Hz}, 1 \mathrm{H}), 2.78(\mathrm{t}, J=7.6 \mathrm{~Hz}, 2 \mathrm{H}), 1.87-1.72(\mathrm{~m}, 2 \mathrm{H}), 1.34-1.21(\mathrm{~m}, 4 \mathrm{H}), 0.83(\mathrm{t}, J=6.9 \mathrm{~Hz}, 3 \mathrm{H}) \mathrm{ppm}$; ${ }^{13} \mathrm{C}$ NMR $\left(100 \mathrm{MHz}, \mathrm{CDCl}_{3}\right): \delta 157.7,142.3,138.6,135.6,130.2,129.5,127.4,119.6,116.9 .110 .4,31.6$, 27.8, 27.5, 22.4, 14.0 ppm; IR: $v_{\max } 3064,2847,2828,1586,1529,1481,1475,1444,1023,998,974,929,784$ $\mathrm{cm}^{-1}$; HRMS (ESI+): $\mathrm{m} / z$ calcd for $\mathrm{C}_{19} \mathrm{H}_{20} \mathrm{~F}_{3} \mathrm{~N}_{2}[\mathrm{M}+\mathrm{H}]^{+}$333.1573; found 333.1574.

1-Benzyl-2-(pyridin-2-yl)-5-(trifluoromethyl)-1H-benzo[d]imidazole $(3\{4,5\})$ : a white solid; $\mathrm{mp}$ 160-161 ${ }^{\circ} \mathrm{C} .{ }^{1} \mathrm{H}$ NMR $\left(400 \mathrm{MHz}, \mathrm{CDCl}_{3}\right): \delta 8.75(\mathrm{~d}, J=7.2 \mathrm{~Hz}, 1 \mathrm{H}), 8.56(\mathrm{~d}, J=7.6 \mathrm{~Hz}, 1 \mathrm{H}), 8.26(\mathrm{~s}$, $1 \mathrm{H}), 7.96\left(\mathrm{dd}, J_{1}=J_{2}=7.8 \mathrm{~Hz}, 1 \mathrm{H}\right), 7.61(\mathrm{~d}, J=8.5 \mathrm{~Hz}, 1 \mathrm{H}), 7.51(\mathrm{~d}, J=8.5 \mathrm{~Hz}, 1 \mathrm{H}), 7.49-7.43(\mathrm{~m}, 1 \mathrm{H})$, 7.41-7.32 (m, 3H), $7.27(\mathrm{~d}, J=7.6 \mathrm{~Hz}, 2 \mathrm{H}), 6.32(\mathrm{~s}, 2 \mathrm{H}) \mathrm{ppm} ;{ }^{13} \mathrm{C}$ NMR $\left(100 \mathrm{MHz}, \mathrm{CDCl}_{3}\right): \delta 151.8,150.0$, $148.8,142.2$, 138.7, 137.0, 136.9, 128.7, 127.6, 126.8, 125.8, 124.9, 124.3, 123.5, 120.3, 117.8, 111.2, 49.2 ppm; IR: $v_{\max } 3066,2938,2854,1621,1584,1492,1447,1429,1045,994,974,930,895,840,792 \mathrm{~cm}^{-1}$; HRMS (ESI+): $m / z$ calcd for $\mathrm{C}_{20} \mathrm{H}_{15} \mathrm{~F}_{3} \mathrm{~N}_{3}[\mathrm{M}+\mathrm{H}]^{+}$354.1213; found 354.1216.

(E)-1-Cyclohexyl-2-styryl-1H-benzo[d]imidazole $(3\{5,9\})$ : colorless oil; ${ }^{1} \mathrm{H}$ NMR $\left(400 \mathrm{MHz}, \mathrm{CDCl}_{3}\right)$ : $\delta 8.01(\mathrm{~d}, J=15.7 \mathrm{~Hz}, 1 \mathrm{H}), 7.82(\mathrm{~d}, J=7.8 \mathrm{~Hz}, 1 \mathrm{H}), 7.67(\mathrm{~d}, J=7.6 \mathrm{~Hz}, 2 \mathrm{H}), 7.59(\mathrm{~d}, J=8.1 \mathrm{~Hz}, 1 \mathrm{H})$, 7.51-7.33 (m, 3H), 7.32-7.16 (m, 3H), 4.51-4.39 (m, 1H), 2.42-2.23 (m, 2H), $2.04(\mathrm{~m}, 4 \mathrm{H}), 1.88(\mathrm{~m}, 1 \mathrm{H})$, 1.65-1.22 (m, 3H) ppm; ${ }^{13} \mathrm{C}$ NMR $\left(100 \mathrm{MHz}, \mathrm{CDCl}_{3}\right): \delta 150.0,143.2,136.9,135.7,133.8,128.4,128.3$, 126.7, 121.6, 121.5, 119.1, 113.6, 55.6, 31.2, 25.7, 24.9 ppm; IR: $v_{\max } 3064,2937,2828,1589,1532,1488$, 1474, 1456, 1023, 986, 973, 929, $782 \mathrm{~cm}^{-1}$; HRMS (ESI+): $\mathrm{m} / z$ calcd for $\mathrm{C}_{21} \mathrm{H}_{23} \mathrm{~N}_{2}[\mathrm{M}+\mathrm{H}]^{+}$303.1856; found 303.1843 .

2-(2-(4-Methoxyphenyl)-1-phenyl-1H-benzo[d]imidazol-5-yl)benzo[d]thiazole $(3\{6,3\})$ : a light yellow solid; mp 209-210 ${ }^{\circ} \mathrm{C} .{ }^{1} \mathrm{H}$ NMR $\left(400 \mathrm{MHz}, \mathrm{CDCl}_{3}\right): \delta 8.43(\mathrm{~s}, 1 \mathrm{H}), 8.06-7.97(\mathrm{~m}, 2 \mathrm{H}), 7.82(\mathrm{~d}$, $J=7.8 \mathrm{~Hz}, 1 \mathrm{H}), 7.50-7.37(\mathrm{~m}, 6 \mathrm{H}), 7.32-7.17(\mathrm{~m}, 4 \mathrm{H}), 6.76(\mathrm{~d}, J=8.2 \mathrm{~Hz}, 2 \mathrm{H}), 3.72(\mathrm{~s}, 3 \mathrm{H}) \mathrm{ppm} ;{ }^{13} \mathrm{C}$ NMR $\left(100 \mathrm{MHz}, \mathrm{CDCl}_{3}\right): \delta 168.9,160.9,154.4,153.9,143.3,139.3,136.8,135.1,131.0,130.1,129.0,128.9$, 127.5, 126.2, 124.9, 123.0, 122.7, 121.9, 121.6, 119.5, 113.9, 110.8, 55.3 ppm; IR: $v_{\max } 3062,2931,2839,1719$, 
$1606,1530,1501,1479,1432,1020,908,835,792 \mathrm{~cm}^{-1}$; HRMS (ESI + ): $m / z$ calcd for $\mathrm{C}_{27} \mathrm{H}_{20} \mathrm{~N}_{3} \mathrm{OS}[\mathrm{M}+\mathrm{H}]^{+}$ 434.1322; found 434.1318.

2-(1-Phenyl-2-(pyridin-2-yl)-1H-benzo[d]imidazol-5-yl)benzo[d]thiazole $(3\{6,5\})$ : a white solid; mp 128-129 ${ }^{\circ} \mathrm{C} .{ }^{1} \mathrm{H}$ NMR $\left(400 \mathrm{MHz}, \mathrm{D}_{2} \mathrm{O}\right): \delta 8.68(\mathrm{~s}, 1 \mathrm{H}), 8.52(\mathrm{~d}, J=4.6 \mathrm{~Hz}, 1 \mathrm{H}), 8.28(\mathrm{~d}, J=8.6 \mathrm{~Hz}, 1 \mathrm{H})$, $8.25-8.17(\mathrm{~m}, 2 \mathrm{H}), 8.03(\mathrm{~d}, J=7.9 \mathrm{~Hz}, 1 \mathrm{H}), 7.88\left(\mathrm{dd}, J_{1}=J_{2}=7.8 \mathrm{~Hz}, 1 \mathrm{H}\right), 7.66-7.56(\mathrm{~m}, 4 \mathrm{H}), 7.53-7.42$ (m, 4H), 7.40-7.34 (m, 1H) ppm; ${ }^{13} \mathrm{C}$ NMR $\left(100 \mathrm{MHz}, \mathrm{D}_{2} \mathrm{O}\right): \delta 168.7,154.3,152.1,149.1,149.0,142.9$, 139.6, 137.3, 136.5, 135.1, 129.4, 129.2, 128.4, 127.3, 126.2, 124.9, 124.7, 123.9, 123.4, 123.0, 121.5, 120.1, 111.4 ppm; IR: $v_{\max } 3058,2931,2856,1726,1588,1496,1442,1018,971,871,790 \mathrm{~cm}^{-1}$; HRMS (ESI+): $m / z$ calcd for $\mathrm{C}_{25} \mathrm{H}_{17} \mathrm{~N}_{4} \mathrm{~S}[\mathrm{M}+\mathrm{H}]^{+} 405.1168$; found 405.1154 .

2-(2-(Furan-2-yl)-1-phenyl-1H-benzo[d]imidazol-5-yl)benzo[d]thiazole $(3\{6,6\})$ : a light yellow solid; mp 143-144 ${ }^{\circ} \mathrm{C} .{ }^{1} \mathrm{H}$ NMR $\left(400 \mathrm{MHz}, \mathrm{CDCl}_{3}\right): \delta 8.51(\mathrm{~s}, 1 \mathrm{H}), 8.14-8.03(\mathrm{~m}, 2 \mathrm{H}), 7.89(\mathrm{~d}, J=7.9 \mathrm{~Hz}$, $1 \mathrm{H}), 7.68-7.57(\mathrm{~m}, 3 \mathrm{H}), 7.55-7.32(\mathrm{~m}, 5 \mathrm{H}), 7.16(\mathrm{~d}, J=8.5 \mathrm{~Hz}, 1 \mathrm{H}), 6.41-6.34(\mathrm{~m}, 1 \mathrm{H}), 6.16(\mathrm{~d}, J=3.4 \mathrm{~Hz}$, 1H) ppm; ${ }^{13} \mathrm{C}$ NMR $\left(100 \mathrm{MHz}, \mathrm{CDCl}_{3}\right): \delta 168.1,153.7,145.1,144.0,143.6,142.8,138.6,134.6,129.9$, 129.6, 128.6, 127.3, 125.7, 124.4, 122.6, 122.4, 121.0, 119.1, 112.4, 111.1, 110.1 ppm; IR: $v_{\max } 3052,2945$, 2828, 1620, 1593, 1499, 1470, 1429, 1026, 973, 911, 861, 822, $762 \mathrm{~cm}^{-1}$; HRMS (ESI+): $\mathrm{m} / \mathrm{z}$ calcd for $\mathrm{C}_{24} \mathrm{H}_{16} \mathrm{~N}_{3} \mathrm{OS}[\mathrm{M}+\mathrm{H}]^{+}$394.1009; found 394.1003.

2-(2-Pentyl-1-phenyl-1H-benzo[d]imidazol-5-yl)benzo[d]thiazole $(3\{6,8\})$ : a light yellow solid; $\mathrm{mp}$ $128-129^{\circ} \mathrm{C} .{ }^{1} \mathrm{H}$ NMR $\left(400 \mathrm{MHz}, \mathrm{CDCl}_{3}\right): \delta 8.43(\mathrm{~s}, 1 \mathrm{H}), 8.12-8.00(\mathrm{~m}, 2 \mathrm{H}), 7.87(\mathrm{~d}, J=7.8 \mathrm{~Hz}, 1 \mathrm{H})$, $7.63-7.50(\mathrm{~m}, 3 \mathrm{H}), 7.45\left(\mathrm{dd}, J_{1}=J_{2}=7.6 \mathrm{~Hz}, 1 \mathrm{H}\right), 7.41-7.29(\mathrm{~m}, 3 \mathrm{H}), 7.16(\mathrm{~d}, J=8.4 \mathrm{~Hz}, 1 \mathrm{H}), 2.78(\mathrm{t}$, $J=7.7 \mathrm{~Hz}, 2 \mathrm{H}), 1.88-1.72(\mathrm{~m}, 2 \mathrm{H}), 1.37-1.21(\mathrm{~m}, 4 \mathrm{H}), 0.84(\mathrm{t}, J=6.8 \mathrm{~Hz}, 3 \mathrm{H}) \mathrm{ppm} ;{ }^{13} \mathrm{C}$ NMR $(100 \mathrm{MHz}$, $\left.\mathrm{CDCl}_{3}\right): \delta 168.5,156.6,153.9,142.5,138.1,135.2,134.6,129.5,128.7,127.9,126.8,125.6,124.3,122.4,121.7$, 121.0, 118.6, 109.6, 31.0, 27.3, 26.8, 21.8, 13.4 ppm; IR: $v_{\max } 3051,2934,2850,1731,1619,1596,1512,1498$, $1465,1457,1441,1006,973,916,868,816,761 \mathrm{~cm}^{-1}$; HRMS (ESI+): $\mathrm{m} / z$ calcd for $\mathrm{C}_{25} \mathrm{H}_{24} \mathrm{~N}_{3} \mathrm{~S}[\mathrm{M}+\mathrm{H}]^{+}$ 398.1685; found 398.1694.

2-\{2-[(E)-2-(4-Methoxyphenyl)ethenyl]-1-phenyl-1H-benzo[d]imidazol-5-yl\}benzo[d]thiazole (3\{6,10\}): a light yellow solid; mp 212-213 ${ }^{\circ} \mathrm{C} .{ }^{1} \mathrm{H}$ NMR $\left(400 \mathrm{MHz}, \mathrm{CDCl}_{3}\right): \delta 8.47(\mathrm{~s}, 1 \mathrm{H}), 8.15-8.06(\mathrm{~m}$, $2 \mathrm{H}), 7.98(\mathrm{~d}, J=15.9 \mathrm{~Hz}, 1 \mathrm{H}), 7.92(\mathrm{~d}, J=7.9 \mathrm{~Hz}, 1 \mathrm{H}), 7.72-7.58(\mathrm{~m}, 3 \mathrm{H}), 7.55-7.34(\mathrm{~m}, 6 \mathrm{H}), 7.27(\mathrm{~d}$, $J=6.7 \mathrm{~Hz}, 1 \mathrm{H}), 6.89(\mathrm{~d}, J=8.5 \mathrm{~Hz}, 2 \mathrm{H}), 6.71(\mathrm{~d}, J=15.9 \mathrm{~Hz}, 1 \mathrm{H}), 3.83(\mathrm{~s}, 3 \mathrm{H}) \mathrm{ppm} ;{ }^{13} \mathrm{C} \mathrm{NMR}(100 \mathrm{MHz}$, $\left.\mathrm{CDCl}_{3}\right): \delta 169.0,160.8,154.6,153.1,143.7,138.8,138.0,135.6,135.3,130.2,129.4,129.3,129.1,128.9$, 127.7, 126.3, 125.0,123.2, 122.7, 121.7, 119.2, 114.5, 111.4, 110.6, 55.5 ppm; IR: $v_{\max } 3061,2937,2838$, $1604,1529,1500,1431,1020,907,889,835,792 \mathrm{~cm}^{-1}$; HRMS (ESI+): $\mathrm{m} / z$ calcd for $\mathrm{C}_{29} \mathrm{H}_{22} \mathrm{~N}_{3} \mathrm{OS}[\mathrm{M}+\mathrm{H}]^{+}$ 460.1478; found 460.1472 .

2-(1-(4-Methoxyphenyl)-2-phenyl-1H-benzo[d]imidazol-5-yl)benzo[d]thiazole $(3\{7,1\})$ : a light yellow solid; mp 187-188 ${ }^{\circ} \mathrm{C} .{ }^{1} \mathrm{H}$ NMR $\left(400 \mathrm{MHz}, \mathrm{CDCl}_{3}\right): \delta 8.55(\mathrm{~s}, 1 \mathrm{H}), 8.16(\mathrm{~d}, J=8.5 \mathrm{~Hz}, 1 \mathrm{H}), 8.09$ $(\mathrm{d}, J=8.1 \mathrm{~Hz}, 1 \mathrm{H}), 7.92(\mathrm{~d}, J=7.9 \mathrm{~Hz}, 1 \mathrm{H}), 7.63(\mathrm{~d}, J=7.2 \mathrm{~Hz}, 2 \mathrm{H}), 7.54-7.45(\mathrm{~m}, 1 \mathrm{H}), 7.44-7.24(\mathrm{~m}$, $7 \mathrm{H}), 7.05(\mathrm{~d}, J=8.8 \mathrm{~Hz}, 2 \mathrm{H}), 3.91(\mathrm{~s}, 3 \mathrm{H}) \mathrm{ppm} ;{ }^{13} \mathrm{C} \mathrm{NMR}\left(100 \mathrm{MHz}, \mathrm{CDCl}_{3}\right): \delta 169.0,159.9,154.5,154.2$, 143.2, 139.8, 135.3, 129.9, 129.8, 129.6, 129.4, 129.1, 128.7, 128.5, 126.3, 125.0, 123.1, 123.0, 121.7, 119.8, 115.3, 111.1, 55.7 ppm; IR: $v_{\max } 3060,2943,2829,1712,1615,1516,1469,1430,1024,914,894,842,796$ $\mathrm{cm}^{-1}$; HRMS (ESI+): $m / z$ calcd for $\mathrm{C}_{27} \mathrm{H}_{20} \mathrm{~N}_{3} \mathrm{OS}[\mathrm{M}+\mathrm{H}]^{+} 434.1322$; found 434.1315.

2-(2-(Furan-2-yl)-1-(4-methoxyphenyl)-1H-benzo[d]imidazol-5-yl)benzo[d]thiazole $(3\{7,6\})$ : a light yellow solid; mp 153-154 ${ }^{\circ} \mathrm{C} .{ }^{1} \mathrm{H}$ NMR $\left(400 \mathrm{MHz}, \mathrm{CDCl}_{3}\right): \delta 8.49(\mathrm{~s}, 1 \mathrm{H}), 8.09(\mathrm{~d}, J=8.5 \mathrm{~Hz}, 1 \mathrm{H})$, $8.06(\mathrm{~d}, J=8.1 \mathrm{~Hz}, 1 \mathrm{H}), 7.90(\mathrm{~d}, J=7.8 \mathrm{~Hz}, 1 \mathrm{H}), 7.56-7.52(\mathrm{~m}, 1 \mathrm{H}), 7.48\left(\mathrm{dd}, J_{1}=J_{2}=7.8 \mathrm{~Hz}, 1 \mathrm{H}\right)$, 7.40-7.31 (m, 3H), 7.19-7.08 (m, 3H), 6.41-6.35 (m, 1H), $6.18(\mathrm{~d}, J=3.4 \mathrm{~Hz}, 1 \mathrm{H}), 3.93(\mathrm{~s}, 3 \mathrm{H}) \mathrm{ppm} ;{ }^{13} \mathrm{C}$ NMR (100 MHz, $\left.\mathrm{CDCl}_{3}\right): \delta 168.7,160.4,154.3,145.9,144.5,144.1,143.0,139.4,135.1,129.1,129.0,128.3$, 126.1, 124.9, 123.1, 123.0, 121.5, 119.5, 115.2, 112.9, 111.6, 110.6, 55.6 ppm; IR: $v_{\max } 3057,2956,2843,1598$, 1534, 1498, 1475, 1456, 1027, 986, 974, 927, $784 \mathrm{~cm}^{-1}$; HRMS (ESI+): $\mathrm{m} / z$ calcd for $\mathrm{C}_{25} \mathrm{H}_{18} \mathrm{~N}_{3} \mathrm{O}_{2} \mathrm{~S}[\mathrm{M}+\mathrm{H}]^{+}$ 424.1114; found 424.1118.

(E)-2-(1-(4-Methoxyphenyl)-2-styryl-1H-benzo[d]imidazol-5-yl)benzo[d]thiazole $(3\{7,9\})$ : a light yellow solid; mp 211-212 ${ }^{\circ} \mathrm{C} .{ }^{1} \mathrm{H}$ NMR $\left(400 \mathrm{MHz}, \mathrm{CDCl}_{3}\right): \delta 8.46(\mathrm{~s}, 1 \mathrm{H}), 8.11(\mathrm{~d}, J=8.5 \mathrm{~Hz}, 1 \mathrm{H}), 8.07(\mathrm{~d}$, $J=8.1 \mathrm{~Hz}, 1 \mathrm{H}), 8.00(\mathrm{~d}, J=16.0 \mathrm{~Hz}, 1 \mathrm{H}), 7.91(\mathrm{~d}, J=7.8 \mathrm{~Hz}, 1 \mathrm{H}), 7.52-7.45(\mathrm{~m}, 3 \mathrm{H}), 7.42-7.31(\mathrm{~m}, 6 \mathrm{H})$, 
$7.24(\mathrm{~d}, J=8.5 \mathrm{~Hz}, 1 \mathrm{H}), 7.14(\mathrm{~d}, J=8.5 \mathrm{~Hz}, 2 \mathrm{H}), 6.83(\mathrm{~d}, J=16.0 \mathrm{~Hz}, 1 \mathrm{H}), 3.95(\mathrm{~s}, 3 \mathrm{H}) \mathrm{ppm} ;{ }^{13} \mathrm{C} \mathrm{NMR}$ $\left(100 \mathrm{MHz}, \mathrm{CDCl}_{3}\right): \delta 159.1,153.3,151.7,142.3,137.9,136.8,134.8,134.1,128.2,128.0,127.8,127.7,126.6$, 126.3, 125.1, 123.8, 121.9, 121.5, 120.5, 118.1, 114.2, 112.5, 109.6, 54.6, 28.6 ppm; IR: $v_{\max } 3051,2957,2835$, $1631,1514,1433,1021,969,912,799 \mathrm{~cm}^{-1}$; HRMS (ESI+): $\mathrm{m} / z$ calcd for $\mathrm{C}_{29} \mathrm{H}_{22} \mathrm{~N}_{3} \mathrm{OS}[\mathrm{M}+\mathrm{H}]^{+} 460.1478$; found 460.1473 .

2-(1-Cyclohexyl-2-phenyl-1H-benzo[d]imidazol-5-yl)benzo[d]thiazole $(3\{8,1\})$ : a light yellow solid; mp 181-182 ${ }^{\circ} \mathrm{C} .{ }^{1} \mathrm{H}$ NMR $\left(400 \mathrm{MHz}, \mathrm{CDCl}_{3}\right): \delta 8.47(\mathrm{~s}, 1 \mathrm{H}), 8.14(\mathrm{~d}, J=12.2 \mathrm{~Hz}, 1 \mathrm{H}), 8.08(\mathrm{~d}$, $J=12.4 \mathrm{~Hz}, 1 \mathrm{H}), 7.89(\mathrm{~d}, J=8.2 \mathrm{~Hz}, 1 \mathrm{H}), 7.73(\mathrm{~d}, J=8.8 \mathrm{~Hz}, 1 \mathrm{H}), 7.66(\mathrm{~d}, J=8.2 \mathrm{~Hz}, 2 \mathrm{H}), 7.59-7.51$ (m, 3H), 7.49-7.43 (m, 1H), 7.39-7.31 (m, 1H), 4.44-4.32 (m, 1H), 2.39-2.163 (m, 2H), 2.07-1.84 (m, 4H), 1.81-1.70 (m, 1H), 1.41-1.24 (m, 3H) ppm; $\left.{ }^{13} \mathrm{C} \mathrm{NMR} \mathrm{(100} \mathrm{MHz,} \mathrm{CDCl}_{3}\right): \delta 168.9,155.3,154.3,143.9,136.1$, $135.1,130.6,129.9,129.4,128.7,128.0,126.2,124.8,122.9,121.7,121.5,120.0,113.0,57.2,31.5,25.9,25.2$ ppm; IR: $v_{\max } 3052,2948,2848,1622,1518,1465,1443,1020,983,931,874,924,809 \mathrm{~cm}^{-1}$; HRMS (ESI+): $\mathrm{m} / \mathrm{z}$ calcd for $\mathrm{C}_{26} \mathrm{H}_{24} \mathrm{~N}_{3} \mathrm{~S}[\mathrm{M}+\mathrm{H}]^{+} 410.1685$; found 410.1679 .

2-(2-Cyclohexyl-1-propyl-1H-benzo[d]imidazol-5-yl)benzo[ $[\underline{d}]$ thiazole $(3\{9,7\})$ : a light yellow solid; mp $164-165^{\circ} \mathrm{C} .{ }^{1} \mathrm{H}$ NMR $\left(400 \mathrm{MHz}, \mathrm{CDCl}_{3}\right): \delta 8.37(\mathrm{~s}, 1 \mathrm{H}), 8.13(\mathrm{~d}, J=8.5 \mathrm{~Hz}, 1 \mathrm{H}), 8.04(\mathrm{~d}, J=8.1 \mathrm{~Hz}$, $1 \mathrm{H}), 7.87(\mathrm{~d}, J=7.9 \mathrm{~Hz}, 1 \mathrm{H}), 7.46\left(\mathrm{dd}, J_{1}=J_{2}=8.1,1 \mathrm{H}\right), 7.42-7.30(\mathrm{~m}, 2 \mathrm{H}), 4.10(\mathrm{t}, J=7.3,2 \mathrm{H}), 2.89-2.73$ $(\mathrm{m}, 1 \mathrm{H}), 2.01-1.75(\mathrm{~m}, 9 \mathrm{H}), 1.49-1.35(\mathrm{~m}, 3 \mathrm{H}), 0.99(\mathrm{t}, J=7.5 \mathrm{~Hz}, 3 \mathrm{H}) \mathrm{ppm} ;{ }^{13} \mathrm{C}$ NMR $\left(100 \mathrm{MHz}, \mathrm{CDCl}_{3}\right)$ : $\delta$ 169.4, 160.8, 154.5, 143.1, 137.1, 135.2, 127.9, 126.2, 124.8, 123.0, 121.6, 121.5, 119.5, 110.0, 45.3, 36.6, 32.2, 26.5, 25.9, 23.6, 11.5 ppm; IR: $v_{\max } 3061,2925,2846,1723,1615,1502,1472,1431,1015,982,918$, $869,819,799 \mathrm{~cm}^{-1}$; HRMS (ESI+): $\mathrm{m} / z$ calcd for $\mathrm{C}_{23} \mathrm{H}_{26} \mathrm{~N}_{3} \mathrm{~S}$ [M+H] ${ }^{+} 376.1842$; found 376.1837.

\section{Conclusions}

In summary, $\mathrm{HfCl}_{4}$ was identified as a highly efficient transition metal Lewis acid catalyst for the synthesis of benzimidazoles from o-phenylenediamines and aldehydes. Our experimental results showed that activated carbon could serve as an excellent solid support for $\mathrm{HfCl}_{4}$ with respect to both catalytic activity and recyclability. The SEM images and EDS spectra of $\mathrm{HfCl}_{4} / \mathrm{C}$ samples along with the desorption experiments revealed that $\mathrm{HfCl}_{4}$ was tightly adsorpted on activated carbon at ambient temperature and partially desorpted in refluxing ethanol. Based on the fact that $\mathrm{HfCl}_{4} / \mathrm{C}$ could catalyze the formation of benzimidazoles at ambient temperature, the catalytic effect of $\mathrm{HfCl}_{4} / \mathrm{C}$ under refluxing conditions should involve both homogeneous and heterogeneous mechanisms. Further application of $\mathrm{HfCl}_{4} / \mathrm{C}$ in parallel synthesis of 1,2-disubstituted benzimidazoles well exemplified its advantages in terms of catalytic efficiency and facile removal from reaction.

Supplementary Materials: The following are available online at http://www.mdpi.com/2073-4344/10/4/436/s1, Figures S1-S38: The NMR spectra of new compounds.

Author Contributions: Contributions: S.-W.D. and Q.S. conceived and designed the experiments; X.-C.P., S.-S.G., and D.-Y.Z. performed the experiments and analyzed the data; S.-W.D. and Q.S. wrote the paper. All authors have read and agreed to the published version of the manuscript.

Funding: This research was funded by National Natural Science Foundation of China (21961013) and Innovation Foundation of JXSTNU (YC2019-X27 for X.-C.P.).

Conflicts of Interest: The authors declare no conflict of interest.

\section{References}

1. Sreerama, R.; Barnali, M.; Balamurali, M.M.; Chanda, K. Synthesis and medicinal applications of benzimidazoles: An overview. Curr. Org. Synth. 2017, 14, 40-60.

2. Carvalho, L.C.R.; Fernandes, E.; Marques, M.M.B. Developments towards regioselective synthesis of 1,2-disubstituted benzimidazoles. Chem. Eur. J. 2011, 17, 12544-12555. [CrossRef] [PubMed]

3. Preston, P.N. Synthesis, reactions, and spectroscopic properties of benzimidazoles. Chem. Rev. 1974, 74, 279-314. [CrossRef]

4. Yadav, G.; Ganguly, S. Structure activity relationship (SAR) study of benzimidazole scaffold for different biological activities: A mini-review. Eur. J. Med. Chem. 2015, 97, 419-443. [CrossRef] [PubMed] 
5. Shah, K.; Chhabra, S.; Shrivastava, S.K.; Mishra, P. Benzimidazole: A promising pharmacophore. Med. Chem. Res. 2013, 22, 5077-5104. [CrossRef]

6. Bansal, Y.; Silakari, O. The therapeutic journey of benzimidazoles: A review. Bioorg. Med. Chem. 2012, 20, 6208-6236. [CrossRef]

7. Alamgir, M.; Black, D.S.C.; Kumar, N. Synthesis, reactivity and biological activity of benzimidazoles. Top. Heterocycl. Chem. 2007, 21, 87-118.

8. Wallace, M.B.; Feng, J.; Zhang, Z.; Skene, R.J.; Shi, L.; Caster, C.L.; Kassel, D.B.; Xu, R.; Gwaltney, S.L., II. Structure-based design and synthesis of benzimidazole derivatives as dipeptidyl peptidase IV inhibitors. Bioorg. Med. Chem. Lett. 2008, 18, 2362-2367. [CrossRef]

9. Nagawade, R.R.; Shinde, D.B. $\mathrm{BF}_{3} \mathrm{OEt}_{2}$ Promoted solvent-free synthesis of benzimidazole derivative. Chin. Chem. Lett. 2006, 17, 453-456.

10. Mayer, J.P.; Lewis, G.S.; McGee, C.; Bankaitis-Davis, D. Solid-phase synthesis of benzimidazoles. Tetrahedron Lett. 1998, 39, 6655-6658. [CrossRef]

11. Kim, S.Y.; Park, K.H.; Chung, Y.K. Manganese (IV) dioxide-catalyzed synthesis of quinoxalines under microwave irradiation. Chem. Commun. 2005, 1321-1323. [CrossRef] [PubMed]

12. Gogoi, P.; Konwar, D. An efficient and one-pot synthesis of imidazolines and benzimidazoles via anaerobic oxidation of carbon-nitrogen bonds in water. Tetrahedron Lett. 2006, 47, 79-82. [CrossRef]

13. Beaulieu, P.L.; Hache, B.; von Moos, E. A practical oxone ${ }^{\circledR}$-mediated, high-throughput, solution-phase synthesis of benzimidazoles from 1,2-phenylenediamines and aldehydes and its application to preparative scale synthesis. Synthesis 2003, 11, 1683-1692. [CrossRef]

14. Sun, Q.; Wu, R.; Cai, S.; Lin, Y.; Sellers, L.; Sakamoto, K.; He, B.; Peterson, B.R. Synthesis and biological evaluation of analogues of AKT (protein kinase B) inhibitor-IV. J. Med. Chem. 2011, 54, 1126-1139. [CrossRef]

15. Sun, Q.; Wang, C.-J.; Gong, S.-S.; Ai, Y.-J.; Sun, H.-B. $\mathrm{Cp}_{2} \mathrm{ZrCl}_{2}$-catalyzed synthesis of 2-aminovinyl benzimidazoles under microwave conditions. Chin. Chem. Lett. 2015, 26, 297-300. [CrossRef]

16. Wu, Y.; Wang, X.; Luo, Y.; Wang, J.; Jian, Y.; Sun, H.; Zhang, G.; Zhang, W.; Gao, Z. Solvent strategy for unleashing the Lewis acidity of titanocene dichloride for rapid Mannich reactions. RSC Adv. 2016, 6, 15298-15303. [CrossRef]

17. Qiu, R.; Xu, X.; Peng, L.; Zhao, Y.; Li, N.; Yin, S. Strong Lewis acids of air-stable metallocene bis(perfluorooctanesulfonate)s as high-efficiency catalysts for carbonyl-group transformation reactions. Chem. Eur. J. 2012, 18, 6172-6182. [CrossRef]

18. Ishihara, K.; Ohara, S.; Yamamoto, H. Direct condensation of carboxylic acids with alcohols catalyzed by hafnium (IV) salts. Science 2000, 290, 1140-1142. [CrossRef]

19. Ishihara, K.; Nakayama, M.; Ohara, S.; Yamamoto, H. Direct ester condensation from a 1:1 mixture of carboxylic acids and alcohols catalyzed by hafnium (IV) or zirconium (IV) salts. Tetrahedron 2002, 58, 8179-8188. [CrossRef]

20. Lundberg, H.; Adolfsson, H. Hafnium-catalyzed direct amide formation at room temperature. ACS Catal. 2015, 5, 3271-3277. [CrossRef]

21. Li, X.-C.; Gong, S.-S.; Zeng, D.-Y.; You, Y.-H.; Sun, Q. Highly efficient synthesis of $\alpha$-aminophosphonates catalyzed by hafnium(IV) chloride. Tetrahedron Lett. 2016, 57, 1782-1785. [CrossRef]

22. Wang, R.; Chen, J.-Z.; Zheng, X.-A.; Kong, R.; Gong, S.-S.; Sun, Q. Hafnium (IV) triflate as a potent catalyst for selective 1-O-deacetylation of peracetylated saccharides. Carbohydr. Res. 2018, 455, 114-118. [CrossRef] [PubMed]

23. Kong, R.; Han, S.-B.; Wei, J.-Y.; Peng, X.-C.; Xie, Z.-B.; Gong, S.-S.; Sun, Q. Highly efficient synthesis of substituted 3,4-dihydropyrimidin-2-(1H)-ones (DHPMs) catalyzed by $\mathrm{Hf}(\mathrm{OTf})_{4}$ : Mechanistic insights into reaction pathways under metal Lewis acid catalysis and solvent-free conditions. Molecules 2019, 24, 364. [CrossRef] [PubMed]

24. Han, S.-B.; Wei, J.-Y.; Peng, X.-C.; Liu, R.; Gong, S.-S.; Sun, Q. Hf(OTf $)_{4}$ as a highly potent catalyst for the synthesis of Mannich bases under solvent-free conditions. Molecules 2020, 25, 388. [CrossRef]

25. Zhang, Z.-H.; Yin, L.; Wang, Y.-M. An expeditious synthesis of benzimidazole derivatives catalyzed by Lewis acids. Catal. Commun. 2007, 8, 1126-1131. [CrossRef]

26. Nagawade, R.R.; Shinde, D.B. Zirconyl (IV) chloride-promoted synthesis of benzimidazole derivatives. Russ. J. Org. Chem. 2006, 42, 453-454. [CrossRef] 
27. Wei, J.-Y.; Han, S.-B.; Peng, X.-C.; Wang, C.-J.; Zeng, D.-Y.; Gong, S.-S.; Sun, Q. Efficient synthesis of fluorinated benzimidazolines, benzoxazolines and benzothiazolines catalyzed by $\mathrm{Hf}(\mathrm{OTf})_{4}$. Heterocycles 2020, 100, 371-382.

28. Trivedi, R.; De, S.K.; Gibbs, R.A. A convenient one-pot synthesis of 2-substituted benzimidazoles. J. Mol. Catal. A Chem. 2006, 245, 8-11. [CrossRef]

29. Sharghi, H.; Aberi, M.; Doroodmanda, M.M. Reusable cobalt(III)-salen complex supported on activated carbon as an efficient heterogeneous catalyst for synthesis of 2-arylbenzimidazole derivatives. Adv. Synth. Catal. 2008, 350, 2380-2390. [CrossRef]

30. Chakrabarty, M.; Karmakar, S.; Mukherjee, R.; Arima, S.; Harigaya, Y. A mild and expedient one-pot synthesis of substituted benzimidazoles in water using a phase-transfer catalyst. Monatsh. Chem. 2009, 140, 375-380. [CrossRef]

(C) 2020 by the authors. Licensee MDPI, Basel, Switzerland. This article is an open access article distributed under the terms and conditions of the Creative Commons Attribution (CC BY) license (http://creativecommons.org/licenses/by/4.0/). 